EPJ Web of Conferences 65, 01003 (2014)

DOI: $10.1051 /$ epjconf/ 20146501003

(C) Owned by the authors, published by EDP Sciences, 2014

\title{
Neutrinoless double beta decay search with SNO+
}

\author{
V. Lozza ${ }^{1, a}$ for the SNO+ Collaboration \\ ${ }^{1}$ IKTP, TU Dresden, Zellescher Weg 19, 01069 Dresden, Germany
}

\begin{abstract}
The SNO+ experiment is the follow up of SNO. The detector is located $2 \mathrm{~km}$ underground in the Vale Canada Ltd.'s Creighton Mine near Sudbury, Ontario, Canada. The active volume of the detector consists of 780 tonnes of Linear Alkyl Benzene (LAB) in an acrylic vessel of $12 \mathrm{~m}$ diameter, surrounded by about 9500 PMTs. The main goal of the SNO+ experiment is the search for neutrinoless double beta decay of ${ }^{130} \mathrm{Te}$. With an initial loading of $0.3 \%$ of natural tellurium (nearly $800 \mathrm{~kg}$ of ${ }^{130} \mathrm{Te}$ ), it is expected to reach a sensitivity on the effective Majorana neutrino mass of about $100 \mathrm{meV}$ after several years of data taking. Designed as a general purpose neutrino experiment, other exciting physical goals can be explored, like the measurement of reactor neutrino oscillations and geo-neutrinos in a geologically-interesting location, watch of supernova neutrinos and studies of solar neutrinos. A first commissioning phase with water filled detector will start at the end of 2013, while the double beta decay phase will start in 2015 .
\end{abstract}

\section{Introduction}

The SNO+ experiment [1] is the follow up of the SNO (Sudbury Neutrino Observatory) experiment [2], where the detector will be filled with 780 tonnes of liquid scintillator. The deep underground location at 6000 m.w.e., and the high radiopurity level of the material used in the detector construction will allow to explore different fields of the neutrino physics.

The primary physical goal of $\mathrm{SNO}+$ is the investigation of the Dirac or Majorana nature of the neutrinos by loading high quantities of double beta decay isotope in the scintillator volume. With an initial loading of $0.3 \%$ of natural tellurium, the aim is to reach a sensitivity of $100 \mathrm{meV}$ on the effective Majorana neutrino mass after several years of data taking.

In addition, the use of liquid scintillator as active volume will lower the detector's threshold allowing to complete the understanding of the solar neutrino fluxes (complementary to SNO) with pep neutrinos (monoenergetic solar neutrinos flux of $1.442 \mathrm{MeV}$ ) and $C N O$ neutrinos. Depending on the background level of $U$ and Th chains and to the purity of the liquid scintillator itself, it will be also possible to detect $p p$ neutrinos.

In order to reuse the SNO Čerenkov detector as a liquid scintillator one, several detector developments were necessary. The most relevant changes are described in section 2. In section 3, the physics program of $\mathrm{SNO}+$ is described.

\section{Detector developments}

The SNO+ active volume consists of 780 tonnes of Linear Alkyl Benzene (solvent, LAB) and $2 \mathrm{~g} / \mathrm{L}$ of 2,5-

\footnotetext{
a e-mail: valentina.lozza@tu-dresden.de
}

diphenyloxazole (fluor, PPO) contained in an acrylic vessel (AV) of $12 \mathrm{~m}$ diameter and $5 \mathrm{~cm}$ thickness. The acrylic sphere is surrounded by about 9500 8" PMTs located on a $18 \mathrm{~m}$ diameter geodesic stainless steel frame (PSUP), for a coverage of $54 \%$. The PSUP and the AV are located in a cavity filled with about 7000 tonnes of ultrapure water (UPW) to shield the detector from the radioactivity coming from the PSUP and the rock. The water volume is monitored by about 100 outward looking PMTs. A radon seal and Urylon liner provide a shield against the mine air. $\mathrm{LAB}+\mathrm{PPO}$ is a suitable scintillator for $\mathrm{SNO}+$ due to its chemical compatibility with the acrylic vessel, the high light yield of about 10000 photons/MeV, the good optical transparency, the low scattering and the fast decay time. Radiopurity requirements for the $\mathrm{SNO}+$ experiment are very close to the one achieved by the Borexino experiment [3]. The level of Th and U in LAB should stay below $10^{-17}$ $\mathrm{g} / \mathrm{g}$. The main background contributions to the search for neutrinoless double beta decay with tellurium are ${ }^{208} \mathrm{Tl}$ and ${ }^{212} \mathrm{BiPo}$ pile-up from the Th chain and ${ }^{214} \mathrm{Bi}$ from the $\mathrm{U}$ chain. To reach the purity goals, a scintillator purification plant similar to the one adopted by Borexino has been designed. LAB will be purified by distillation, water extraction, nitrogen and steam stripping, and the use of metal scavenger adsorption columns [4]. The scintillator purification plant is currently being installed underground and has been thoroughly cleaned. In addition, tagging techniques are under development to constrain the remaining background. The water purification plant has also been upgraded.

Heavy metal loading in LAB, such as neodymium and tellurium, is also possible. However, the optical properties degrade increasing the amount of metal dissolved and a good compromise between loading level, stability and 


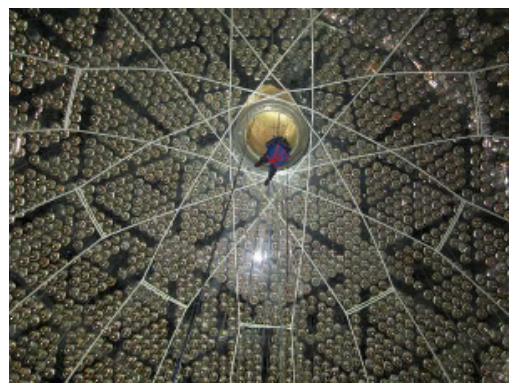

Figure 1. Hold-down rope net system. The system has been fully installed and the ropes are pre-tensioned.

light output must be found.

On the detector development side, the main change for the transition from a heavy water Čerenkov detector to a liquid scintillator one is the detector's holding system. LAB has a density of $0.86 \mathrm{~g} / \mathrm{cm}^{3}$, which leads to a large buoyant force on the acrylic vessel from the surrounding light water. To keep the AV in place, a hold-down rope net system has been installed in 2012 (see fig. 1). The ropes are anchored to the bottom of the cavity (see fig. 2). To reduce the radioactivity contamination, since the ropes are in contact with the detector, they are made of a high purity material (Tensylon) with a low ${ }^{40} \mathrm{~K}$ content.

To reduce the radioactivity background, the inner AV surface and part of the external AV surface have been thoroughly washed with UPW. This process has the aim to remove the dust deposited on the surface after the drain of the SNO detector.

The SNO electronics and data acquisition have been upgraded and tested in 2012 since a high event rate with respect to SNO is expected due to the lower threshold and the higher light yield. Damaged PMTs have been repaired or replaced.

The calibration system of the detector is being upgraded as well to have it compatible with the liquid scintillator. A new set of radioactive calibration sources is under development: $\operatorname{AmBe}(\mathrm{n}, \gamma),{ }^{16} \mathrm{~N}(\gamma),{ }^{24} \mathrm{Na}(\gamma),{ }^{48} \mathrm{Sc}(\gamma),{ }^{57,60} \mathrm{Co}(\gamma)$, ${ }^{90} \mathrm{Y}(\beta)$ and a ${ }^{8} \mathrm{Li}$ - based Čerenkov source.

To reduce the risk of detector contamination due to the sources' deployment, an in-situ optical calibration system based on LEDs and LASER sources has been designed and partly installed. The installation will be completed by the end of 2013.

The current water level inside the cavity is 9 feet (at the bottom of the PSUP). The water filling will be completed by the end of 2013 .

\section{Physics goals}

Three different operation phases are planned for the SNO+ detector. During the initial phase, the detector will be filled with ultrapure water (UPW). The detector response will be checked and a first energy calibration performed. In addition, the search for the nucleon decay will be conducted. The UPW will then be replaced by 780 tonnes of liquid scintillator. The detector response and background (both

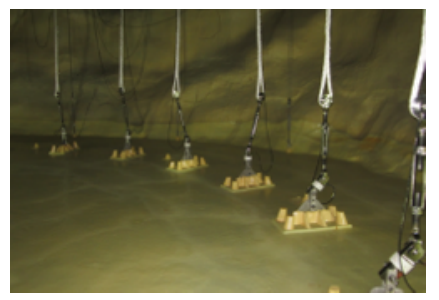

Figure 2. A total of 20 anchors are used to hold the ropes.The anchors are currently under the water level.

internal and external) will be studied. A calibration campaign will also be made. After the pure liquid scintillator phase, natural tellurium at $0.3 \%$ loading will be deployed. The primary goal will be the search for neutrinoless double beta decay of ${ }^{130} \mathrm{Te}$. However, searches for geo and reactor anti-neutrinos will be simultaneously done. The detector will also watch for supernovae neutrinos. Following the initial double beta phase, there is the potential for a new pure scintillator phase, during which measurements of pep and $C N O$ solar neutrinos would be made.

\section{$3.10 \gamma 2 \beta$}

Nowadays, one of the most interesting questions in the field of neutrino physics is whether neutrinos are Dirac or Majorana particles. The test of the Majorana nature of neutrinos can be performed in Double-Beta-Decay (DBD) experiments [5]. The DBD is characterized by a nuclear process where the $\mathrm{Z}$ number changes by 2 units while the atomic mass, $\mathrm{A}$, does not change. It can be seen as two simultaneous $\beta$-decays and happens only for even-even nuclei, and only if single $\beta$-decay is forbidden or strongly suppressed. If neutrinos are Majorana particles, the two neutrons decay into two protons via a neutrino exchange with only two electrons emitted in the final state. In this case the two electron spectrum is no more a continuum, but a peak at an energy equal to the reaction Q-value. The quantity measured is the effective Majorana neutrino mass. A large number of experiments are currently searching for the neutrinoless double beta decay, or are under construction. In the $\mathrm{SNO}+$ experiment the double beta isotope is directly loaded in the detector. Even if, as a liquid scintillator experiment, $\mathrm{SNO}+$ will suffer from a poor energy resolution, this may be compensated for by the high level loading, the low background and the background rejection techniques (like the application of a fiducial volume cut). The SNO+ experiment has initially investigated ${ }^{150} \mathrm{Nd}$ as potential candidate. However, after a first internal suggestion in fall 2011 and nearly two years of investigation and development, the collaboration has decided to focus on ${ }^{130} \mathrm{Te}$. ${ }^{130} \mathrm{Te}$ has a high isotopic abundance of $34.08 \%$ and a Q-value of $2.53 \mathrm{MeV}$. Its double beta decay halflife of $7.0 \cdot 10^{20}$ y [6] is nearly a factor 100 longer than the ${ }^{150} \mathrm{Nd}$ one, which will reduce the $2 v 2 \beta$ signal, one of the main irreducible backgrounds for the $0 v 2 \beta$ searches. In addition, the Te-loaded scintillator cocktail, differently from $\mathrm{Nd}$, does not show any strong absorption line in the 
region where the PMTs are sensitive $(350 \mathrm{~nm}-550 \mathrm{~nm})$. Bench-top measurements have demonstrated that the intrinsic yield is nearly $10^{4}$ photons/MeV for $0.5 \%$ loading, higher than the one obtained with the Nd-loaded scintillator.

A method for loading a high concentration of tellurium in the liquid scintillator has been developed by the SNO+ collaboration. Loading up to $0.3 \%$ has been proven to be stable over one year. Research and developments to increase the loading and improving the optical properties are ongoing.

A multi-pass purification method has been developed by the collaboration to remove $U$ and Th backgrounds. A reduction factor larger than $10^{4}$ has been achieved in a two step pass.

The target levels for $\mathrm{U}$ and $\mathrm{Th}$ contaminations in the Teloaded cocktail are set to $3 \cdot 10^{-15} \mathrm{~g}_{U} / \mathrm{g}_{\text {Cocktail }}$ and $3 \cdot 10^{-16}$ $\mathrm{g}_{T h} / \mathrm{g}_{\text {Cocktail }}$. Additional backgrounds can be produced by the cosmogenic induced activation of natural tellurium during the handling on surface. Production rates have been studied for isotopes with a Q-value larger than $2 \mathrm{MeV}, \mathrm{T}_{1 / 2}$ larger than 20 days and that can be produced by spallation reactions on tellurium. A purification strategy has been developed by the SNO+ collaboration with the aim to reduce the cosmogenic induced background to a negligible level. Tellurium will be firstly purified on surface (double pass recrystallization) achieving a reduction factor larger than $10^{4}$. A second stage double-pass purification will then be done underground to remove short and medium lived isotopes that could have been re-produced after the first purification on surface. The second stage purification will achieve a factor larger than 100. Additional 6 months cooling down underground will finally reduce the cosmogenic background to a negligible level. Due to the depth of the $\mathrm{SNO}+$ experiment the in-situ activation has been estimated to be negligible.

The expected energy spectrum for a $0.3 \%$ Te-loading, 2 years of running with a fiducial volume cut of $3.5 \mathrm{~m}$ is shown in fig. 3. The plot is obtained with $99.99 \%$ and $97 \%$ tagging efficiencies for ${ }^{214} \mathrm{BiPo}$ and ${ }^{208} \mathrm{Tl}$ respectively, a rejection factor of 50 for the in-window ${ }^{212} \mathrm{BiPo}$. For reference, the expected neutrinoless double beta decay signal, assuming an effective Majorana neutrino mass of $200 \mathrm{meV}$ [7][8], is shown.

Through these various techniques, $\mathrm{SNO}+$ is expected to reach a sensitivity to $0 v 2 \beta$-decay at the level lower than $100 \mathrm{meV}$.

\subsection{Solar neutrinos}

After the precise measurement achieved by SNO on the ${ }^{8} \mathrm{~B}$ solar neutrino flux, and the one of Borexino on ${ }^{7} \mathrm{Be}$ neutrinos, the next important steps are the detections of the pep, $p p$ and $C N O$ neutrinos. The $\mathrm{SNO}+$ experiment, during a pure liquid scintillator phase, will be sensitive to energies also below $3.5 \mathrm{MeV}$ (the SNO threshold), which will allow a measurement of the pep neutrinos, momoenergetic 1.442 $\mathrm{MeV}$ neutrinos. This energy is in the vacuum-matter transition region. An accurate measurement of the neutrino survival probability will improve the precision on the oscillation parameters and the sensitivity to alternative models of neutrino mixing.

If background contaminations at the Borexino level are reached, thanks to the unique underground location which will reduce the background due to the muon induced ${ }^{11} \mathrm{C}$ of a factor 100 with respect to Borexino level, a precise measurement, with an uncertainty of about $6.5 \%$, after two year of data taking, can be achieved.

A measurement of $C N O$ neutrinos tests the solar metallicity [9]. The main source of background for the measurement of $C N O$ neutrinos is ${ }^{210} \mathrm{Bi}$. Its shape is very close to the searched signal, making the discrimination difficult. In addition, the inner surface of the AV has been exposed to mine air over its lifetime from construction to after SNO was drained. Mine air is rich in radon that has implanted on the surface. The long lived daughter of radon $\left({ }^{210} \mathrm{~Pb}\right)$ is still present on the AV surface and can leach into the liquid scintillator increasing the background level of ${ }^{210} \mathrm{Bi}$. Although the purification system has the capability to remove radon daughters that leach into the scintillator, a high leaching rate can make the pep or $C N O$ neutrino measurement difficult. Additional mitigation steps might be needed, like, for instance, enhancing in-situ purification or draining the detector and sanding the AV surface, or deploying an internal bag. A background tagging method may also be defined, in order to separate the signal and ${ }^{210} \mathrm{Bi}$.

\subsection{Geo and reactor anti-neutrinos}

$\mathrm{SNO}+$ can measure anti-neutrino events via the inverse beta decay interaction. The decay process can be tagged using the delayed coincidence between the signal from the positron annihilation (prompt) and the neutron capture on protons (about $220 \mu$ s later).

Anti-neutrinos are produced by the $\mathrm{U}$ and Th long lived nuclides in the Earth crust and mantel. In SNO+ it will be possible to measure the anti-neutrino flux coming from the Earth with high enough statistics to be comparable with the measurement previously done by KamLAND in 2005 [10] and by Borexino [11]. The measurement will contribute to a multi-site measurement which will help to understand the heat production of the Earth, revealing the uranium and thorium content of the deep Earth. This is possible since the composition of the Canadian Shield (the geological region around $\mathrm{SNO}+$ ), is different from the one of Japan or Italy, where KamLAND and Borexino are respectively placed. In $\mathrm{SNO}+$ about 29 geo anti-neutrinos events are expected in one year of data taking [12].

$\mathrm{SNO}+$ can also measure anti-neutrinos from reactors. In particular the detector is located near three main reactors which creates two effective distinct baselines. This makes distinct feature in the energy spectrum of the detected antineutrinos. The consequence is that $\mathrm{SNO}+$ has the potential to make a constraint on $\Delta m_{12}^{2}$ competitive with the one of KamLAND [13] even if the expected flux is a factor 5 smaller. 


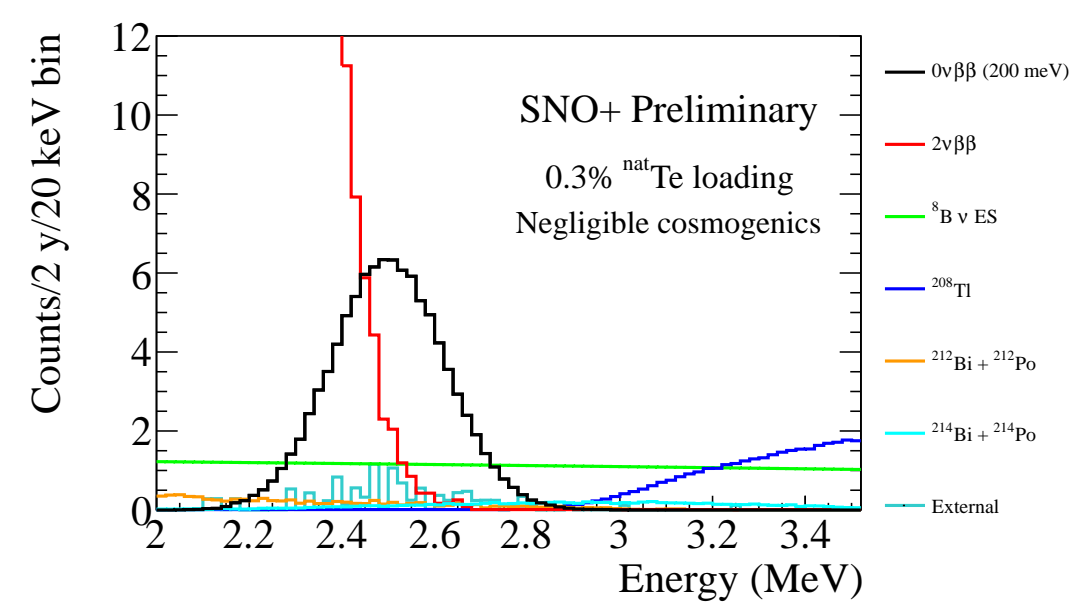

Figure 3. Expected energy spectrum (average profiles) for the SNO+ $0 v 2 \beta$-decay search. The spectrum is obtained for 2 years of running, a fiducial volume (FV) cut of $20 \%$ and $0.3 \%$ Te-loading $\left(800 \mathrm{~kg}\right.$ of $\left.{ }^{130} \mathrm{Te}\right)$. A $99.99 \%$ efficient tag for ${ }^{214} \mathrm{BiPo}, 97 \%$ efficient tag for ${ }^{208} \mathrm{Tl}$ and a factor 50 rejection for ${ }^{212} \mathrm{BiPo}$ are used. External backgrounds are reduced by the FV cut and by a likelihood ratio. Negliglible cosmogenic induced background is assumed. The expected $0 v 2 \beta$-decay signal, assuming an effective Majorana neutrino mass of $200 \mathrm{meV}$, is also shown for comparison [7][8].

\subsection{Supernovae neutrinos}

SNO+ will be part of the the Supernova Early Warning System (SNEWS). For a Galactic supernova of $10^{53} \mathrm{erg}$ at $10 \mathrm{kpc}, \mathrm{SNO}+$ expects to detect more than 100 neutrinos via charge-current interactions with an energy threshold of $0.2 \mathrm{MeV}$. Furthermore, supernova neutrinos can be detected via neutral-current interactions on ${ }^{12} \mathrm{C}$ and proton scattering with a comparable number above $0.2 \mathrm{MeV}$. Via neutral current interactions it would be possible to gain information on the total flux of all neutrino flavours.

\section{Conclusion}

The SNO+ experiment is a 780 tonnes liquid scintillator based experiment that will search for the neutrinoless double beta decay of ${ }^{130} \mathrm{Te}$. With a $0.3 \%$ loading and about 2 years of data taking, $\mathrm{SNO}+$ will be able to set a limit on the effective Majorana neutrino mass $<100 \mathrm{meV}$. In addition, due to the low energy threshold, $\mathrm{SNO}+$ will be able to measure low energy solar neutrinos, like pep and CNO neutrinos. Geo and reactor anti-neutrinos will also be measured. Furthermore, $\mathrm{SNO}+$ will be part of the Supernova early warning system. Several changes have been made to the detector in order to reuse the SNO vessel. A new hold-down ropes system has been installed, the DAQ and detector electronics have been upgraded to deal with the higher event rate. SNO+ is currently nearly completition with about 9 feet of ultra pure water in the detector cavity. The water fill will be completed by the end of the year with the detector running end of 2013 beginning of 2014. The Te-loading phase is foreseen for early 2015.

\section{Acknowledgement}

The work was supported by the Deutsche Forschungsgemeinschaft.

\section{References}

[1] M.C. Chen, Nucl. Phys. B (Proc. Suppl.) 145, 65-68 (2005)

[2] The SNO Collaboration, J. Boger et al., Nucl. Instrum. Methods A 449, 172-207 (2000)

[3] M. Pallavicini, Nucl. Phys. B (Proc. Suppl.) 217, 101-106 (2011)

[4] R. Ford, M. Chen, O. Chkvorets, D. Hallman and E. Vazquez-Jauregui, AIP Conf. Proc. 1338, 183-194 (2011)

[5] K. Zuber, Contemporary Phys. 45, 491-502 (2004)

[6] The NEMO-3 Collaboration, R. Arnold et al., Phys. Rev. Lett. 107, 062504 (2011)

[7] J. Kotila and F. Iachello, Phys. Rev. C 85, 034316 (2012)

[8] J. Barea, J. Kotila, F. Iachello, Phys. Rev. C 87, 014315 (2013)

[9] W.C. Haxton and A.M. Serenelli, Ap. J. 687, 678691 (2008)

[10] T. Araki et al., Nature 436, 499-503 (2005)

[11] The Borexino Collaboration, G. Bellini et al., Phys. Lett. B 687, 299-304 (2010)

[12] M.C. Chen, Earth, Moon, and Planets 99, 221-228 (2006)

[13] The KamLAND Collaboration, A. Gando et al., Phys. Rev. D 83, 052002 (2011) 